\title{
Comparative Analysis of Different Prescriptive Formulae Used in the Evaluation of Real Ear Insertion Gain for Digital Hearing Aids
}

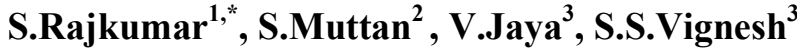 \\ ${ }^{1}$ Department of ECE, Kings Engineering College, Chennai-602117, India \\ ${ }^{2}$ Department of ECE, CEG, Anna University, Chennai-600025, India \\ ${ }^{3}$ Speech pathologist \& Audiologist, Madras Medical college \&Rajiv Gandhi Government Hospital, Chennai \\ *Corresponding Author: srk1670@yahoo.com
}

Copyright (C) 2013 Horizon Research Publishing All rights reserved.

\begin{abstract}
Various prescriptive procedures have been developed, for calculating the real ear insertion gain of a digital hearing aid based either on loudness equalization or speech intelligibility. But the problem for audiologists lies in selecting an appropriate procedure for particular types of hearing impairment. The aim of this work is to analyze various linear and nonlinear prescriptive procedures, by performing various audiological investigations on hearing impaired subjects, so as to select an optimum procedure for a specific type of impairment. An audiological test was carried out on 237 hearing impaired subjects in the age group of 23 to 75 years, and they were given appropriate hearing aids with gain recommendations, using different formulae by audiologists. The degrees of success of the recommended formulae were analyzed, based on the speech discrimination score. Subjects with mild and moderate losses were found satisfied with the gain suggestions of linear prescriptive procedures, in addition to procedures based on loudness equalization NAL-RP (National Acoustics Laboratory-Revised Profound), POGO II (prescription of Gain Output), and DSL (Desired Sensation Level). Subjects with sensorineural loss were satisfied only with the gain recommendations of non linear prescriptive procedures NAL-NL1 (National Acoustics Laboratory-Non Linear), NAL-NL2 and DSL I/O (Desired Sensation Level Input/Output). An appropriate prescriptive procedure for a specific audiogram could be successfully suggested in future based on the results obtained from the total population on whom the study was carried out. In the case of those subjects who were not satisfied with the suggested procedure, a revised formula would be arrived at, by suitably varying the gains by the audiologists
\end{abstract}

Keywords Real Ear Insertion Gain, Audiogram, Gain-frequency Response, Prescriptive Procedure, Loudness Equalization and Speech Intelligibility

\section{Introduction}

Audiology is very much concerned with hearing aid selection, and the interaction of the hearing impaired person for satisfaction. Initially Watson and Knudsen [1] and then Lybarger [2], suggested a way to prescribe parameters for hearing aids, based on audiometric measurements. In the earlier days prescriptive procedures were not a serious concern because of technological limitations. The development of Behind-The-Ear (BTE), In-The-Ear (ITE) hearing aids, and the probe microphone, made prescriptive fitting techniques essential. Once the person's audiological data are obtained, the clinician must be cautious in suggesting the appropriate prescriptive fitting technique with the specific type of hearing loss and hearing aid circuitry. Earlier, prescriptive hearing aid selection had been done only with linear hearing instruments. DSL I/O [3], IHAFF [4], and FIG6 [5] procedures focuses on nonlinear hearing aid circuits. The appropriate choice of the algorithm determines the success of the prescriptive hearing aid fitting. The challenge for the audiologists is in choosing the right prescriptive fitting technique, and modifies it to satisfy the subject.

It is essential that every subject is given a comprehensive audiological evaluation, which includes pure tone air and bone conduction audiometry, speech audiometry, speech at its most comfortable level (MCL), and speech at its uncomfortable level (UCL). Most prescriptive procedures require only pure tone test results for the REIG calculations. Also, some programmable systems require special equipment and specific measurement protocols, as part of their fitting process. It is reasonable to assess the inter octave frequencies, especially 1500 and $3000 \mathrm{~Hz}$ because not only do some prescriptive formulae calculate the gain and output at these inter octave frequencies, but the hearing impairment could change somewhat over a full octave. In addition, irrespective of the fitting approach, it appears that there should be strong clinical contraindications to a binaural 
fitting, before clients are directed to the monaural use of hearing aids $[6,7]$.

Hawkins's approaches to the specification of gain and frequency response can be categorized in several ways. Researchers decide systematically what they want the hearing aid to do and why; then specific formulae are developed to achieve the stated goal [8]. Usually, these formulae are based upon research that supports a particular approach to the hearing aid fitting question. Cornelisse et al., reported that the prescription as a frequency-specific gain function that can be prescribed for each individual with hearing impairment, on the basis of audiometric data [3]. These gain algorithms can be subdivided into two classes: formulae that make use of threshold audiometric data and formulae that incorporate suprathreshold audiometric data in deriving electro acoustic prescriptions for listeners with hearing impairment. While Byrne states that the gain and frequency response requirements appear to be about equally predictable from the threshold or suprathreshold measures, particular formulae may or may not accurately reflect the specific relationships between the audiological measure and the amplification parameters [9]. The specifics of the prescriptive formula generation and detailed discussion can be found in Humes and Halling [10] and McCandless [11].

The objectives of prescriptive approaches are i) to provide an appropriate gain to achieve normal hearing, ii) to present an average speech spectrum at a comfortable level to the ear, iii) to provide the maximum dynamic range, iv) to provide signals to restore equal loudness function, v) to provide aided speech signals at MCL in the speech frequencies, vi) to provide gain based on the size and shape of the dynamic range, and vii) to provide gain based upon the discomfort level [12]. The prescriptive technique allows for an organized, systematic approach to hearing aid fitting, that maximizes the user benefit in a short length of time. A detailed discussion of the advantages and limitations of the various popular and obscure prescriptive fitting approaches can be obtained from various sources [8-11].This discussion will further focus upon the two most often used approaches for linear instruments, as well as present the more recent prescriptive methods specifically designed for nonlinear hearing aids, including National Acoustic Laboratory Revised (NAL-R), Prescription of Gain and Output (POGO) (POGO II), Desired Sensation Level Input/output (DSL I/O), Independent Fitting Forum (IHAFF) and FIG6.

The NAL and POGO procedures have been available since 1976 and 1983, respectively. The revised versions, NAL-R (1986) and POGO II (1988) created greater accuracy and utility, producing great popularity among prescriptive procedure users. Since the existing most popular methods were developed from research with linear instruments [13], nonlinear circuits that adjust according to the input signal are not well reflected in these linear approaches. The rise of the use of conventional and programmable nonlinear hearing aid circuits, has created a focus on new prescriptive methodologies to accommodate nonlinearity, and assist clinicians in their ability to fit these products easily and accurately. Specifically, approaches that are the result of this research include the DSL I/O [3], IHAFF [14], FIG6 [5, 15], NAL-NL1 [16] and NAL-NL2 [17]. Analyzing all the parameters and standards used in the prescriptive procedures, the present work considers the appropriate procedure for a specific hearing impairment. The Speech Discrimination Score (SDS) is obtained, by incorporating the suitable prescriptive procedure suggestions in the digital hearing aid. The procedure which gives the best score will be considered as an appropriate procedure for that type of hearing loss.

\section{Material \& Methods}

\subsection{Linear Prescriptive Procedures}

\subsubsection{National Acoustics Laboratory Revised (NAL-R)}

Byrne and Tonnisson [18] described the National Acoustics Laboratory (NAL) formula and later Byrne and Dillon [19] revised it as NAL-R. The NAL prescribes a frequency response that optimizes the potential for the understanding of speech after the hearing aid volume control has been set to the wearer's preferred level. Speech optimization is to maximize the available speech signal, averaged over a wide frequency range. It would most likely be achieved if all the frequency bands of speech contributed equally to the loudness of the signal. This procedure has not achieved the goal of amplifying all speech bands to equal loudness at a comfortable listening level, or to the Most Comfortable Level (MCL). Generally, too little gain was provided to a low frequency signal compared to the mid and high frequency signals and to provide too much variation in the frequency response slopes for the variation in the subjects' audiogram slopes [18]. To solve this problem and make effective use of the NAL procedure, Byrne and Dillon proposed a revised version of the NAL procedure, the NAL-R.

The NAL-R specifically refined and optimized the energy available for certain hearing losses in all speech bands, particularly the low frequency band between 500 and 1000 $\mathrm{Hz}$, and reduced the calculation from the half slope or the half gain rule to the third slope or the one-third gain rule. Although the calculation for the threshold is a third slope, the NAL-R procedure requires a calculation of the three frequency average, i.e., pure tone average. The three frequency average calculation increases at a rate of 46 percent of the three frequency audiometric average, as given in equation 1 . The gain for different frequencies was adjusted by an amount, in accordance with the shape of the long term average spectrum (LTASS), so that less gain will be given to the frequencies where speech is more intense (low frequencies). The shape of the gain frequency response may be obtained by subtracting the shape of the speech spectrum from the normal equal loudness curve and adding 0.46 times the shape of the hearing threshold curve. The NAL formula includes the reserve gain of $10 \mathrm{~dB}$, so that the hearing aid gain is measured with the coupler at full volume control, and 
later it is used at the comfortable level by the subject [20]. NAL-R formula

$$
\text { Insertion Gain }(\mathrm{IG})=\mathrm{X}+0.31 * \mathrm{H}_{\mathrm{T}}+\mathrm{C}
$$

where,

$$
\mathrm{X}=0.15 * \mathrm{PTA}
$$

Pure Tone Average $(\mathrm{PTA})=\left(\mathrm{H}_{\mathrm{T}} 500+\mathrm{H}_{\mathrm{T}} 1 \mathrm{k}+\mathrm{H}_{\mathrm{T}} 2 \mathrm{k}\right) / 3$

$\mathrm{H}_{\mathrm{T}}$ is the threshold of hearing for the respective frequencies $\mathrm{C}$ is the correction factor. It is different for each octave frequency as shown in table 1 .

$\mathrm{H}_{\mathrm{T}} 500, \mathrm{H}_{\mathrm{T}} 1 \mathrm{k}, \mathrm{H}_{\mathrm{T}} 2 \mathrm{k}$ are measured hearing thresholds at $500 \mathrm{~Hz}, 1 \mathrm{KHz}$ and $2 \mathrm{KHz}$ respectively

Table 1. Correction factor for various octave frequencies in NAL- R formula

\begin{tabular}{|c|c|c|c|c|c|c|c|}
\hline $\begin{array}{c}\text { Frequency } \\
(\mathrm{Hz})\end{array}$ & 250 & 500 & 1000 & 2000 & 3000 & 4000 & 6000 \\
\hline $\mathrm{C}$ in dB & -17 & -8 & 1 & -1 & -2 & -2 & -2 \\
\hline
\end{tabular}

The specific NAL-R formula for the calculation of real-ear insertion gain (REIG) of a person with flat $40 \mathrm{~dB}$ loss, is shown in fig 1. It provides very small gain for frequencies up to $1 \mathrm{KHz}$, and almost a constant gain for frequencies beyond $2 \mathrm{KHz}$.

\subsubsection{NAL-RP (Revised, Profound) formula}

The NAL-R formula did not provide equal loudness for subjects with steeply sloping losses in the high frequency region. It provides better satisfaction for subjects with mild or moderate loss, whereas for people with severe and profound loss, two modifications of the NAL-R formula have been suggested, because these subjects required additional gain and less high frequency emphasis [21, 22]. Modification 1 suggested an increase in the required gain, i.e., $66 \%$ of the hearing loss instead of $46 \%$ if the PTA exceeds $60 \mathrm{~dB}$ [8]. The additional low frequency emphasis is required to maximize the speech intelligibility based on the hearing threshold at $2 \mathrm{KHz}$. The second modification increases the gain in the low, and reduces the gain in the high frequencies, if the degree of hearing loss at $2000 \mathrm{~Hz}$ exceeds $90 \mathrm{~dB}$. This adjustment alters the hearing aid response to the necessary changes in the high and low frequency regions, to accommodate a person with severe hearing impairment, because these subjects require more low frequency energy for power and less high frequency energy to reduce feedback problems. The required correction factor $\mathrm{A}$ for different frequencies is shown in table 2 . The gain calculation of NAL $-\mathrm{RP}$ was performed, as given in equation 2.

$$
\text { Insertion Gain }(\mathrm{IG})=\mathrm{X}+0.31 * \mathrm{Ht}+\mathrm{A}
$$

where

$$
\begin{array}{ll}
X=0.15^{* P T A} & \text { for PTA }<60 \\
X=0.15^{* P T A}+0.2(\text { PTA-60) } & \text { for PTA }>60
\end{array}
$$

$\mathrm{A}$ is correction factor
Table 2. NAL -RP adjustment for hearing losses when the hearing threshold at $2 \mathrm{KHz}$ exceeds $90 \mathrm{~dB}$

\begin{tabular}{|c|c|c|c|c|c|c|c|c|c|}
\hline \multirow{2}{*}{$\begin{array}{c}\text { HL at } 2 \mathrm{kHz} \\
\mathrm{dBHL}\end{array}$} & \multicolumn{7}{|c|}{ Frequency in KHz. } \\
\cline { 2 - 10 } & 0.25 & 0.5 & 0.75 & 1 & 1.5 & 2 & 3 & 4 & 6 \\
\hline 95 & 4 & 3 & 1 & 0 & -1 & -2 & -2 & -2 & -2 \\
\hline 100 & 6 & 4 & 2 & 0 & -2 & -3 & -3 & -3 & -3 \\
\hline 105 & 8 & 5 & 2 & 0 & -3 & -5 & -5 & -5 & -5 \\
\hline 110 & 11 & 7 & 3 & 0 & -3 & -6 & -6 & -6 & -6 \\
\hline 115 & 13 & 8 & 4 & 0 & -4 & -8 & -8 & -8 & -8 \\
\hline 120 & 15 & 9 & 4 & 0 & -5 & -9 & -9 & -9 & -9 \\
\hline
\end{tabular}

\subsection{Prescription of Gain and Output II (POGO II) Procedure}

The objective of the POGO procedure introduced in 1983 was to present a practical consideration about the gain preferences of persons with hearing impairment [23]. An additional concern was to outline an approach that presented the gain and output as critical characteristics of the prescription [12]. Essentially, POGO was based on the Lybarger's half gain Rule; because of the greater intensity of speech at low frequencies, a correction factor was suggested for the low frequency signals, to facilitate better speech understanding ability called as speech intelligibility in that region. The insertion gain at each frequency is half of the hearing loss at that frequency, plus the low frequency conversion factor shown in equation 3.

$$
\text { Insertion Gain }(\mathrm{IG})=0.5 * \mathrm{H}_{\mathrm{T}}+\mathrm{k}
$$

$\mathrm{H}_{\mathrm{T}}$ is the hearing threshold at each octave frequency. $\mathrm{k}$ is the low frequency conversion factor, equal to $-10 \mathrm{~dB}$ and $-5 \mathrm{~dB}$ for $250 \mathrm{~Hz}$ and $500 \mathrm{~Hz}$ respectively. For the remaining frequencies it was not suggested; hence, it is zero.

The gain suggestions by the POGO procedure for an audiogram of flat $40 \mathrm{~dB}$ hearing loss are shown in fig2. It provides small gain for frequencies up to $0.5 \mathrm{KHz}$ and constant gain for frequencies above $1 \mathrm{KHz}$. The POGO formula gives a satisfactory performance for subjects having hearing loss up to $80 \mathrm{~dB}$ only, in all the frequencies. So, to give satisfaction to subjects with severe and profound hearing loss, the formula was revised in 1988, and named as POGO II formula. For them, the gain is increased by $1 \mathrm{~dB}$ for every $1 \mathrm{~dB}$ increase in hearing loss. In POGO II, the gain formula is modified when the hearing loss was greater than $65 \mathrm{~dB}$ HL. The gain is further increased by half the amount that the hearing loss exceeds $65 \mathrm{~dB}$ [24]. The gain suggestions by the POGO II prescriptive procedure for a patient with $40 \mathrm{~dB}$ hearing loss is compared with the NAL-R suggestions, as shown in figure 1 .

POGO II formula

$$
\text { Insertion Gain }(\mathrm{IG})=0.5 * \mathrm{H}_{\mathrm{T}}+\mathrm{k} \quad \text { for } \mathrm{H}_{\mathrm{T}}<65
$$$$
\text { Insertion Gain }(\mathrm{IG})=0.5 * \mathrm{H}_{\mathrm{T}}+\mathrm{k}+0.5 *\left(\mathrm{H}_{\mathrm{T}}-65\right) \text { for } \mathrm{H}_{\mathrm{T}}>65
$$ 


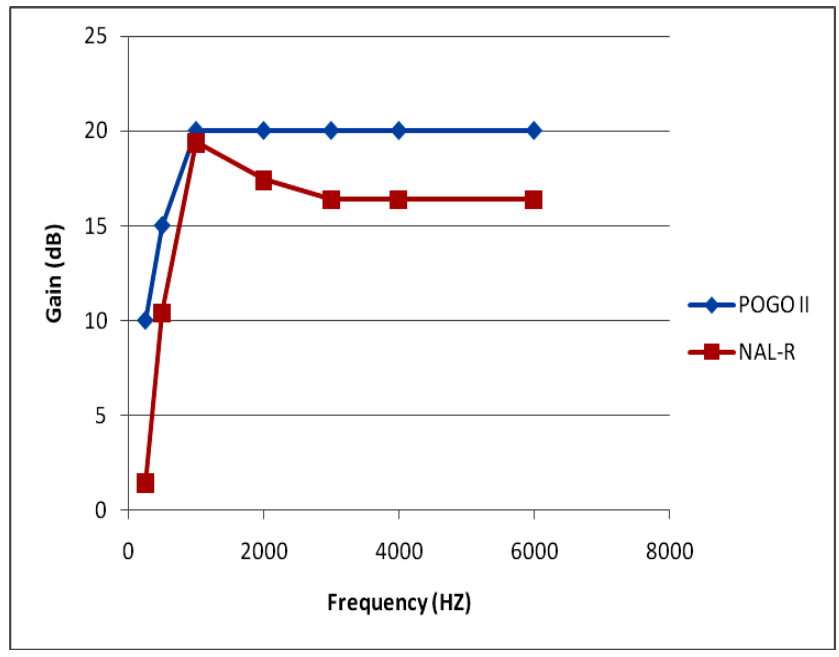

Figure 1. Gain suggestions of NAL-R and POGO II for a subject with flat $40 \mathrm{~dB}$ hearing loss.

\subsection{Desired Sensation Level (DSL) Procedure}

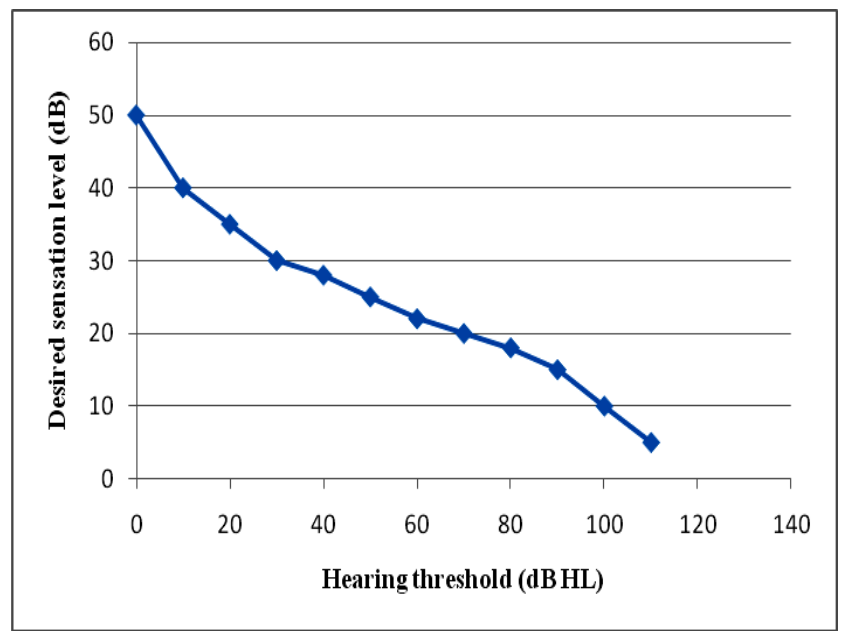

Figure 2. Sensation level targets for the Desired sensation level method as a function of hearing threshold at $1 \mathrm{KHz}$.

The DSL method differs from the previous procedures NAL-RP and POGO II in several aspects. First, it prescribes real ear aided gain rather than real ear insertion gain. Real ear aided gain is the SPL near the ear drum minus the SPL at some reference point outside the head. Real ear insertion gain is the effective gain obtained by the signal because of the hearing aid usage. Second, it does not require average correction factors when used for infants and young children. Lastly, it does not make the speech equally loud in every frequency region, and for different types of hearing loss, the procedure specifies a desired sensation level, as shown in figure.2. The speech signal should be amplified to a comfortable level of loudness. The most comfortable listening level is approximated midway between the threshold of audibility and the UCL level. The amplified targets are defined as the output targets (not gain targets). The gain required to reach these targets is calculated, by subtracting 1/3-octave band level of LTASS, SPL in the sound field from the MCL target. As the hearing threshold increases, the target sensation level decreases, because a subject with profound hearing loss has only a small dynamic range between the threshold and discomfort. In this procedure, the target real ear aided gain (REAG) is calculated using the desired sensation level. For each frequency, the REAG is equal to the desired sensation level plus the hearing threshold, minus the short term maximum speech levels in the field for speech at an overall level of 70dB SPL. The DSL REAG has been converted into insertion gain, by subtracting the adult average real ear unaided gain curve.

\subsection{Non Linear Prescriptive Procedures}

In the case of non linear prescriptive procedures the gain is calculated by considering the gain-frequency response of various input levels. The average gain and shape of the frequency response will vary with the input level of the signal. The required compression ratios and thresholds are easily read from the set of Input/output (I/O) curves. The Filters are used to form the individual channels in a multichannel hearing aid. Filter characteristics are read from the set of gain-frequency responses.

\subsubsection{Desired Sensation Level Input / Output (DSL I/O) Procedure}

The DSL I/O procedure is evolved from the DSL procedure first used as a prescriptive method for children [25, 26]. The Objective is to achieve the desired sensation level of the amplified signal for multiple level inputs. DSL [I/O] can also be used as an effective method of achieving Loudness Equalization. Apply an acoustic transform to the input signal, such that the hearing impaired listener's perceived loudness matches the perceived loudness of a normal hearing person, across frequencies. Comelisse et al. [3] indicated that their purpose was to develop a device-independent formula for the specification of the electro acoustic characteristics of a personal hearing aid or, theoretically, the ideal amplified output for a range of input levels. They summarized the DSL I/O approach as a series of mathematical equations shown in table 3 , that describe the relationship between the input level of a signal delivered to a hearing aid, and the output level produced by the hearing aid. The DSL [I/O] program provides frequency-specific output targets for multiple input signal levels, based upon speech, not on tones. The I/O approach divides the input dynamic range into three regions: 1) input levels below a compression threshold, or Imin; 2) input levels that will exceed the compression threshold when amplified, or Imax; and, 3) the area between these two limits. If the input signal level is equal to or less than Imin, linear gain is applied to the signal (i.e., below compression threshold). If the signal level is equal to or greater than Imax, the output is limited to Omax (output limiting). For input signals between 'Imin and Imax, The I/O formula applies compression to the signal. The I/O formula is frequency-specific, (i.e., the $\mathrm{I} / \mathrm{O}$ is described for 
each frequency band). I/O formula is also device-independent; that is, the formula was not designed for any particular model, but rather was designed to be applicable to the class of wide dynamic range compression hearing aids. Furthermore, the I/O approach can be applied to fit either a single channel or a multichannel compression device. The Specification of frequency-specific gain and maximum output are sufficient to describe the electro acoustic characteristics of a linear gain hearing aid. The available selection procedures are inadequate for the compression hearing aid, because these algorithms only specify one gain value. Their approach was to describe the frequency-specific I/O characteristic of a compression device.

Table 3. Desired Sensation Level Input / Output Formula for calculating Real Ear Insertion Gain

\begin{tabular}{|c|c|}
\hline Input conditions & $\begin{array}{c}\text { Output (Real Ear Insertion Gain } \\
\text { Formula })\end{array}$ \\
\hline I $<$ I min $=($ Linear Gain $)$ & $\mathrm{O}=$ O min I min I $)$ \\
\hline $\begin{array}{c}\text { I min }<\text { I }<\text { Imax (Linear } \\
\text { Compression })\end{array}$ & $\begin{array}{c}\mathrm{O}=(\mathrm{I}-\text { Imin } / \text { Imax-Imin }) *(O \\
\text { max-Omin })+ \text { Omin }\end{array}$ \\
\hline I $>$ I max (Output Limiting) & $\mathrm{O}=$ Omax \\
\hline
\end{tabular}

\subsubsection{Calculation of DSL I/O}

The hypothetical data used in this example are for an individual with $50 \mathrm{~dB}$ hearing loss at $1000 \mathrm{~Hz}$, as shown in figure 3. The input SPL sound field level noted as dBSF is plotted along the abscissa, and the output SPL ear canal level noted as $\mathrm{dBEC}$ is plotted along the ordinate.

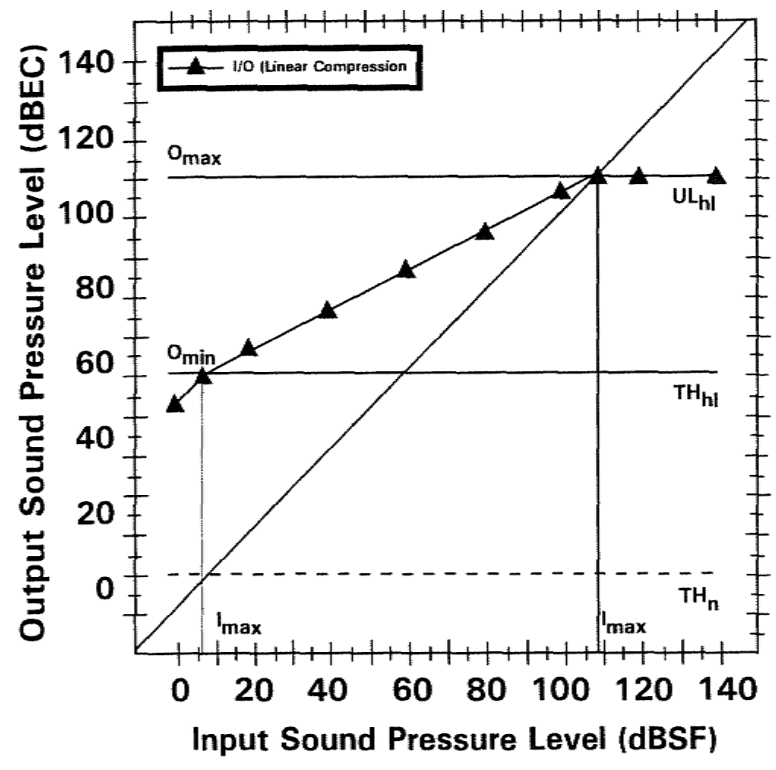

Figure 3. The I/O linear compression functions for the $50-\mathrm{dB}$ hearing loss at $1000 \mathrm{~Hz}$.

The diagonal line indicates the transfer function from the unaided input level to the unaided output level. When a horizontal line and vertical line intersect along the diagonal line, then the horizontal and vertical correspond to equivalent levels at the two measurement points, $\mathrm{dBEC}$ and
dBSF. The DSL I/O must be plotted for each frequency usually 250, 500, 750, 1000, 1500, 2000, 3000, 4000, and $6000 \mathrm{~Hz}$. The standard hearing-impaired auditory dynamic ranges are indicated with horizontal lines. In this example, Imax is equal to $107.4 \mathrm{dBSF}$, and Imin is equal to $7.0 \mathrm{dBSF}$. The aided input dynamic range (DR a) equals ULhI minus THn which is 100.4 for this example [3]. The DSL I/O is a complex formula that makes use of many variables at different frequencies simultaneously, to facilitate the accurate calculation of the specific parameters of the hearing aid fitting. The use of a computer program requires the clinician to enter the threshold data, and automatically calculates the variables and presents ideal $\mathrm{I} / \mathrm{O}$ curves for a particular type of hearing impairment. In the DSL version 5, compression was prescribed to meet the goals of providing audibility, and comfortable loudness of important speech cues, given the gain limits of hearing instruments, and the limited dynamic range of the individual hearing instrument user. This differs from the loudness normalization approach in the previous versions of the DSL algorithm.

\subsubsection{Independent Hearing Aid Fitting Forum (IHAFF)}

A group of researchers, engineers, and clinicians formed the IHAFF, with the aim of developing a standardized comprehensive hearing aid fitting protocol, to assist the audiologists in the selection and fitting of nonlinear and programmable hearing aids, with adjustable wide range dynamic compression. The IHAFF protocol assumes that if an appropriate hearing aid operates to restore the sensation of loudness perception over a wide bandwidth, the wearer of the hearing aid will have a high probability of achieving maximum speech recognition ability. The primary goals of the IHAFF protocol are to provide amplification, so that soft speech is perceived as soft, conversational level speech is perceived as comfortable and loud speech, and involves the use of warble tones presented to the client at a minimum of two frequencies, typically $500 \mathrm{~Hz}$ and $3000 \mathrm{~Hz}$. The client judges their loudness on a scale of seven categories of loudness (Very soft, Soft, Comfortable but slightly soft, Comfortable, Comfortable but slightly loud, Loud but OK, Uncomfortably loud). The IHAFF used a particular type of loudness scaling, called as the contour test. The Visualization of the Input/output Locator Algorithm (VIOLA) is the heart of the IHAFF procedure, as shown in figure 4 , which assists in the management of the variables involved in hearing aid circuit selection. It calculates the relationship between the overall speech input levels for soft, average, and loud speech at the hearing aid microphone, and the user's loudness judgments for warble tones (27). The individual loudness judgment categories, along with an I/O graph noting the input between 30 and $110 \mathrm{~dB}$ in $10 \mathrm{~dB}$ increments, at the hearing aid microphone versus output of the hearing aid, measured in HA-1, 2 cc coupler. Ultimately, the VIOLA software allows the clinician to enter different combinations of the overall gain at an input level of $40 \mathrm{~dB}$, output, and compression characteristics such as the threshold knee point and compression ratio, maximum output, overall 
gain at $60 \mathrm{~dB}$, and slope characteristics at the frequencies for which the loudness judgments were measured.

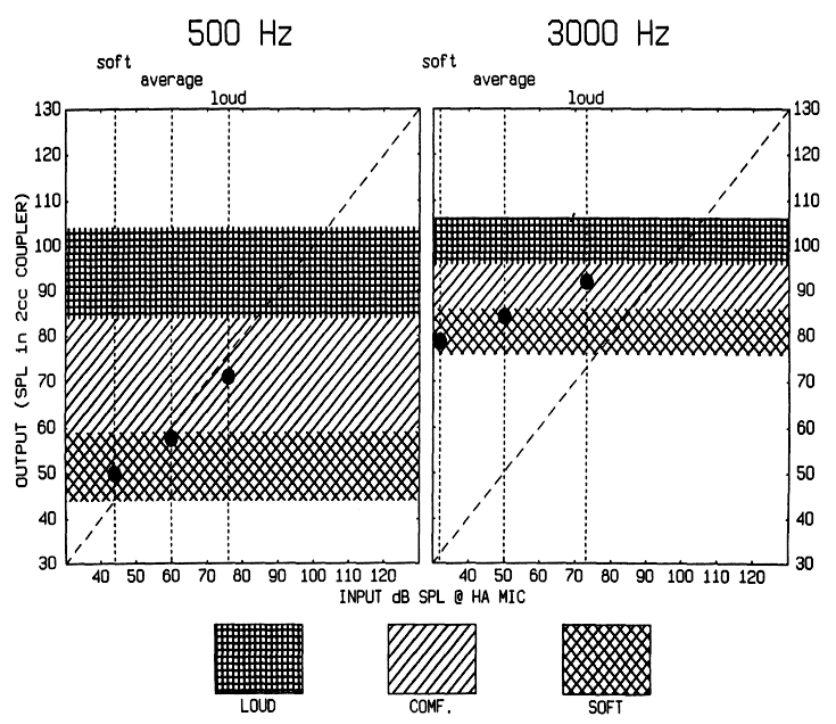

Figure 4. Visual input/output locator algorithm (VIOLA)

The IHAFF suggested that the compression threshold be chosen in the range of 40 to $45 \mathrm{~dB}$ SPL. The calculated I/O curve is then displayed and evaluated by the clinician, for compliance with the target. If the instrument chosen does not meet the target, then the characteristics of devices can be changed, or the instrument can be changed, if it is the conventional type. The VIOLA software screen will predict 2 cc coupler gain for hearing aids. Although the IHAFF is still being researched, it promises to be a precise, predictive formula for nonlinear instruments.

\subsubsection{FIG 6 Procedure}

The FIG 6 Procedure is a loudness-based fitting formula, designed to accommodate the types of hearing losses, described earlier by Killion and Fikret-Pasa (28); indeed the name of this approach is derived from the loudness growth concept presented in Figure 6 of that article. Killion has added a loudness-based fitting formula, to the array of prescriptive fitting procedures. In its current form, it is a spreadsheet approach to estimate the level-dependent gain and frequency response of nonlinear hearing aids (5).

Table 4. REIG calculation in FIG 6 procedure

\begin{tabular}{|c|c|}
\hline \multirow[b]{2}{*}{ Type A } & Gain for low level sounds \\
\hline & $\begin{array}{c}\text { 1. } \mathrm{G}=0 \text { for } 0 \text { to } 20 \mathrm{dBHL} \\
\text { 2. } \mathrm{G}=\mathrm{HL}-20 \text { for } 20 \text { to } 60 \mathrm{~dB} \mathrm{HL} \\
\text { 3. } \mathrm{G}=\mathrm{HL}-20-.5^{*}(\mathrm{HL}-60) \text { for } \mathrm{HL}>=60 \mathrm{~dB} \text {. }\end{array}$ \\
\hline \multirow[b]{2}{*}{ Type B } & Gain at MCL \\
\hline & $\begin{array}{c}1 \mathrm{G}=0 \text { for } 0 \text { to } 20 \mathrm{dBHL} \\
\text { 2. } \mathrm{G}=0.6 *(\mathrm{HL}-20) \text { for } 20 \text { to } 60 \mathrm{~dB} \mathrm{HL} \\
\text { 3. } \mathrm{G}=0.8 * \mathrm{HL}-23 \text { for } \mathrm{HL}>=60 \mathrm{~dB} .\end{array}$ \\
\hline \multirow[b]{2}{*}{ Type C } & Gain for high-level sounds \\
\hline & $\begin{array}{l}\text { 1. } \mathrm{G}=0 \text { for } 0 \text { to } 40 \mathrm{dBHL} \\
\text { 2. } \mathrm{G}=0.1 *(\mathrm{HL}-40) \mathrm{A} 1.4 \text { for } \mathrm{HL}>=40 \mathrm{~dB} \text {. }\end{array}$ \\
\hline
\end{tabular}

Since these aids can change their gain and frequency response depending upon the input level, the FIG6 can be utilized to calculate the gain and frequency response for low-level $45 \mathrm{~dB}$ SPL, moderate-level $65 \mathrm{~dB}$ SPL, and high level $95 \mathrm{~dB}$ SPL sounds (29). The formula for the FIG 6 procedure is presented in table 4 . The FIG6 procedure is a software program that asks the clinician to enter the person's audiogram, and graphically presents a calculation of REIG, and also it prescribes the required coupler response and compression ratios for low \& high input levels and low \& high frequencies.. If a hearing aid is to be ordered for this, the FIG6 automatically calculates the REIG, and the appropriate average coupler response for flat insertion gain (CORFIG) for the BTE, ITE, ITC and CIC hearing aids, as shown in table 5. To convert the REIG to a $2 \mathrm{cc}$ coupler gain, the above numbers are subtracted from the REIG. To convert the $2 \mathrm{cc}$ coupler gain to the predicted REIG, the above numbers are added to the 2cc coupler gain (30).

Table 5. Average CORFIGs for BTE, ITE, ITC Hearing Aids (Hawkins 1992b)

\begin{tabular}{|c|c|c|c|c|c|c|c|}
\hline \multirow{2}{*}{ Hearing Aid Type } & \multicolumn{7}{|c|}{ Frequency in kHz. } \\
\cline { 2 - 8 } & .25 & .5 & 1 & 2 & 3 & 4 & 6 \\
\hline BTE & -3 & -2 & -2 & -7 & -10 & -10 & 0 \\
\hline ITE & 0 & 0 & 0 & -2 & -6 & 0 & 3 \\
\hline ITC & 0 & 0 & 0 & -2 & -3 & 3 & 7 \\
\hline
\end{tabular}

\subsubsection{NAL-NL1 procedure}

The NAL-NL1 is a threshold-based procedure that prescribes the gain-frequency responses for different input levels, or the compression ratios at different frequencies, in wide dynamic range compression hearing aids. The aim of the NAL-NL1 is to maximize speech intelligibility for any input level of speech above the compression threshold, while keeping the overall loudness of speech at or below normal overall loudness. The formula is derived from optimizing the gain-frequency response for speech presented at 11 different input levels to 52 different audiogram configurations, on the basis of two theoretical formula (31.16). The NAL-RP procedure can be applied to non linear hearing aids to the input level of 65-70 dB SPL. At higher or lower input levels; the response varies on the compression rationale selected by the audiologist or the hearing aid designer. Noise reduction and loudness normalization are the important needs. The main objective is to maximize the speech intelligibility. To derive the gain-frequency response at each input level, two theoretical models are used. The first model is based on the speech intelligibility index method. The second model is used for calculating loudness. Hearing thresholds and speech spectrum levels input to the ear after amplification are required for both the models. The two inputs to the process are the input speech spectrum and level, and the audiogram for which a prescription is required. The output is the prescription expressed as a gain-frequency response. The two input parameters are entered into two feedback loops, 
which operate in tandem to optimize the gain-frequency response. One loop uses an intelligibility model to find the gain-frequency response that maximizes speech intelligibility. The calculated loudness is compared to the loudness that would be perceived by a normal-hearing person listening to the same input speech spectrum and level. If the loudness calculated for the audiogram exceeds the normal-hearing loudness, the overall gain is decreased. The NAL-NL1 procedure is based on a complex equation that specifies the gain at each standard 1/3-octave frequency from $125 \mathrm{~Hz}$ to $8000 \mathrm{~Hz}$. At each frequency, the gain depends on the threshold at that frequency, the PTA value, slope of the audiogram from $500 \mathrm{~Hz}$ to $2 \mathrm{KHz}$, and the overall level of the broad band signal with long term speech spectrum. For various speech input level, the gain at each frequency was systematically varied, until the calculated speech intelligibility was maximized by maintaining the loudness level. This procedure displays the results, either as gain curves at different input levels or I/O curves at different frequencies. For multi channel hearing aids, the procedure recommends cross over frequencies, compression thresholds, compression ratios, and gains for 50,65 and $80 \mathrm{~dB}$ SPL input levels. The evaluation of the NAL-NL1 showed that the prescribed overall gain was slightly too high for adults, particularly, for higher input levels, and slightly too low for lower input levels for children.

\subsubsection{NAL-NL2 Procedure}

The NAL-NL2 is the second generation of prescription procedures from The National Acoustic Laboratories (NAL), for fitting wide dynamic range compression (WDRC) instruments. Like NAL-NL1, NAL-NL2 aims at making speech intelligible, and the overall loudness comfortable. This aim is mainly driven by a belief, that these factors are most important for hearing aid users, but is also driven by the fact that less information is available about how to adjust the gain to optimize other parameters that affect prescription, such as localization, tonal quality, detection of environmental sounds, and naturalness $(17,32)$. The objective is achieved by combining a speech intelligibility model and a loudness model in an adaptive computer-controlled optimization process. Adjustments have further been made to the theoretical component of NAL-NL2 directed by empirical data collected during the past decade with NAL-NL1. The adaptive optimization technique used in the NAL-NL2 procedure is shown in fig 5 . One loop uses an intelligibility model to find the gain-frequency response that maximizes speech intelligibility. The second loop therefore uses a loudness model to calculate the loudness, which would be perceived by the hearing-impaired person with the selected gain-frequency response [33, 34]. The adaptive process was used to derive the optimal gain-frequency responses for 240 audiograms, covering a wide range of severity and slopes, each at seven speech input levels from 40 to $100 \mathrm{~dB}$ SPL. First, the intelligibility model, which is a revised version of the speech intelligibility index (SII) formula, was updated. The difference between the original SII formula and the speech intelligibility model used to derive the NAL-NL1 and NAL-NL2, is in the audibility factor. In the speech intelligibility model used in the optimization procedure, an effective audibility factor has been introduced, which takes into account the fact, that as the hearing loss gets more severe, less information is extracted from the speech signal, even when it is audible above the threshold. Second, constraints to the selected gain were applied, such that no compression was introduced for speech presented below $50 \mathrm{~dB}$ SPL, and no gain was prescribed at frequencies below $50 \mathrm{~Hz}$ and above $16 \mathrm{kHz}$. The NAL-NL2 prescribes relatively more gain across low and high frequencies, and less gain across mid frequencies than the NAL-NL1 $[35,36]$.

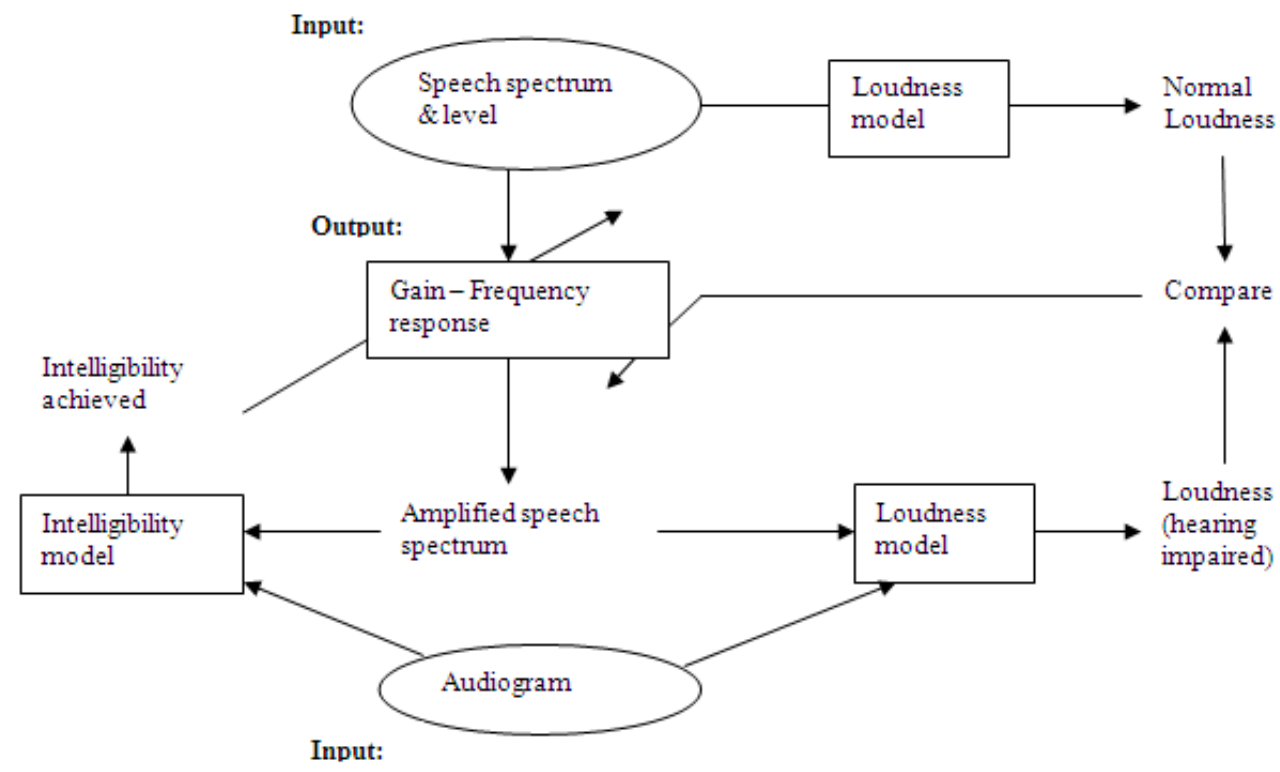

Figure 5. Adaptive optimization process in NAL-NL2 procedure 


\section{Results and Discussion}

\subsection{Comparison of Prescriptive Procedures}

We have considered 237 test subjects with hearing loss over a period of three years; of them 46 were female and 191 were male, in ages ranging between 23 and 75 years. They were suggested prescriptive gain for their digital hearing aid, using different prescriptive procedures. Among these subjects, 116 subjects suffered from conductive hearing loss, and the rest of them were with sensorineural hearing loss. Every procedure uses its distinct formula, which leads to different prescriptive gain suggestions for similar types of audiograms, or patients with the same degree of hearing loss. In the early periods of evaluation, the prescriptive procedures NAL-RP, POGO II and DSL are used. The comparisons of gain suggestions by these procedures were done. As an example a patient suffering from moderately steep sloping loss is shown in table 6 .

Table 6. Audiometric test results of a subject with moderately steep sloping loss

\begin{tabular}{|c|c|c|c|c|c|c|c|c|}
\hline $\begin{array}{c}\text { Frequency } \\
(\mathrm{Hz})\end{array}$ & 250 & 500 & 1000 & 1500 & 2000 & 3000 & 4000 & 6000 \\
\hline $\begin{array}{c}\text { Hearing } \\
\text { threshold } \\
(\mathrm{dB})\end{array}$ & 25 & 30 & 55 & 65 & 80 & 85 & 90 & 90 \\
\hline
\end{tabular}

The insertion gains suggested for the subject for different frequencies using these procedures is plotted as shown in figure 6 . The gain suggestions in the low frequencies up to 1 $\mathrm{KHz}$ by these prescriptive procedures are almost the same. In the high frequency region, the gain suggested by the POGO II and DSL method is almost the same, and distinguished from the NAL-RP procedure. The NAL-RP suggested lower gain for the high frequency region, when compared to the other methods to increase speech intelligibility.

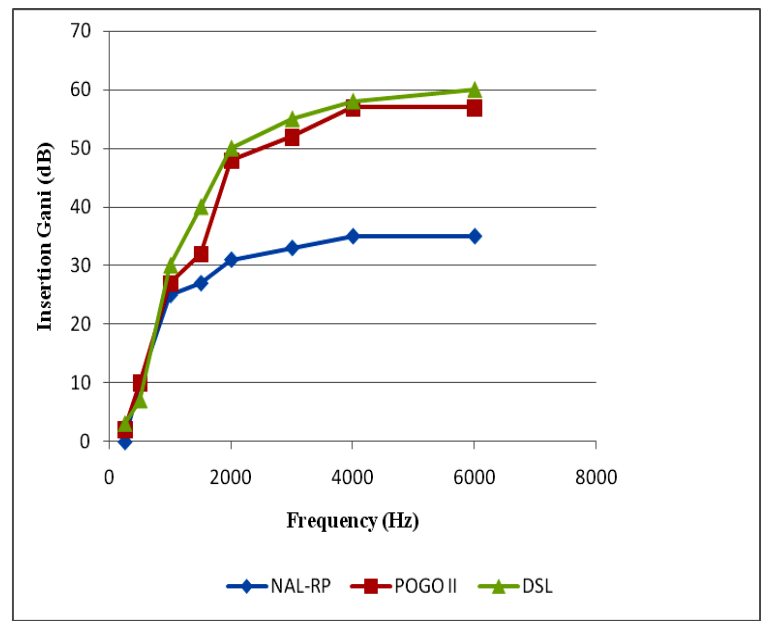

Figure 6. Comparison of insertion gain suggestions by prescriptive procedures NAL-RP, POGO II and DSL.

The difference in the gain prescriptions can be thought of as a difference in the average gain plus a difference in the shape of the frequency response. In the case of the NAL-RP prescriptive procedure the suggested insertion gain at $3 \mathrm{KHz}$ and $4 \mathrm{KHz}$ is less than the target gain, because of the restricted range of response slopes that most hearing aids have in the range of 2 to $4 \mathrm{KHz}$. As the hearing threshold of an individual increases above $60 \mathrm{dBHL}$, the usefulness of high frequency speech information decreases remarkably. In the case of some subjects, the increase in the high frequency information even to moderate audibility decreases the speech intelligibility. Speech intelligibility is measured using the speech intelligibility index (SII). Hearing loss desensitization is the decreased ability of the impaired ear to extract information from a signal that is audible. Subjects with mild and moderate loss obtained satisfaction, by the gain suggestions by these prescriptive procedures, whereas the subjects who suffered from moderately severe and profound loss, evaluated using these procedures, were not satisfied by the gain suggestions.

In the subsequent evaluation period non linear prescriptive procedures like the DSL I/O, IHAFF, FIG6, NAL-NL1 and NAL-NL2 were also used for the gain suggestions. Differences between the responses prescribed by different prescriptive procedures are verified for subjects with different types of hearing loss. The Gain prescriptions by the NAL-NL1, DSL I/O, IHAFF and FIG 6 procedures for a subject with $40 \mathrm{~dB}$ flat hearing loss, for different input levels such as 50, 65 and $80 \mathrm{~dB}$ SPL, are shown in figure7a-c.

The IHAFF and DSL I/O procedures prescribe more or less the same gain for all the frequencies for this subject, who has flat hearing loss. The FIG 6 procedure prescribes a constant level of gain for all frequencies for different input levels. The NAL - NL 1 prescribes less gain for low frequency signals compared to all other procedures. For mid and high frequency regions the gain preferred is more than that prescribed by the other procedures. The NAL-NL1 procedure normally will not suggest high gain for the frequencies with high loss, because of the decreased ability of the ear to extract information at those frequencies. For a low input level, the NAL-NL1 may not prescribe much gain to get audibility at the frequencies where the loss is high, or the frequencies at which speech is the least important for intelligibility. The DSL I/O prescribes a more appropriate gain-frequency response for mild level inputs than the NAL formula. Keidser and Grant (1999) compared two channel Wide Dynamic Range Compression (WDRC) hearing aids, fitted with the NAL-NL1 procedure, and loudness normalization achieved using IHAFF protocols. Preferences in the speech identification score obtained in the lab condition and real time environment, favored the NAL-NL1 procedure. Jenstad et al (2000) performed experiments with WDRC hearing aids, fitted with DSL I/O normalized loudness, more closely than that achieved using linear hearing aids fitted with the linear DSL prescription, tested with narrow and broadband stimuli. Subjects with sensorineural loss obtained satisfactory performance with the digital hearing aid usage, with the gain suggestions of prescriptive procedures based on speech intelligiblity like NAL-NL1 and NAL-NL2. 


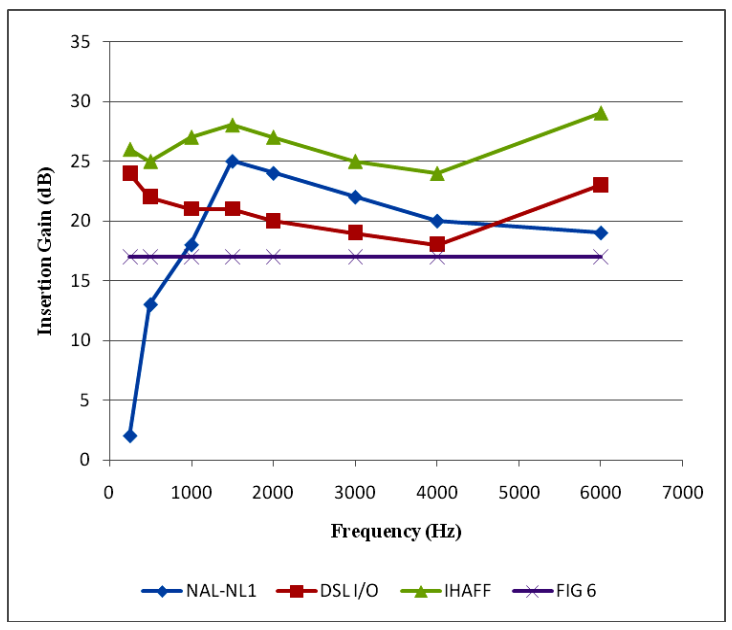

Figure 7a. Insertion gain at input level $50 \mathrm{~dB}$ SPL for each of the four selection procedures of a person with flat $40 \mathrm{~dB}$ hearing loss.

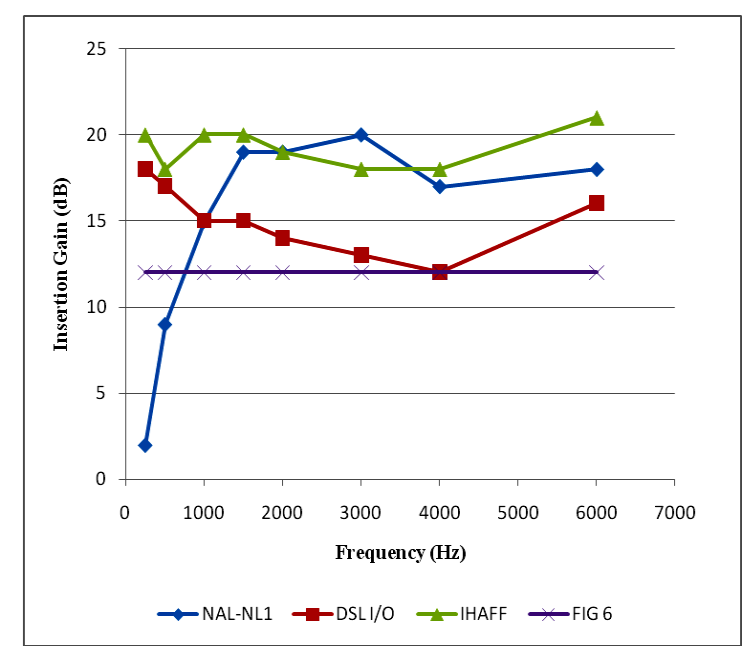

Figure 7b. Insertion gain at input level $65 \mathrm{~dB}$ SPL for each of the four selection procedures of a person with flat $40 \mathrm{~dB}$ hearing loss.

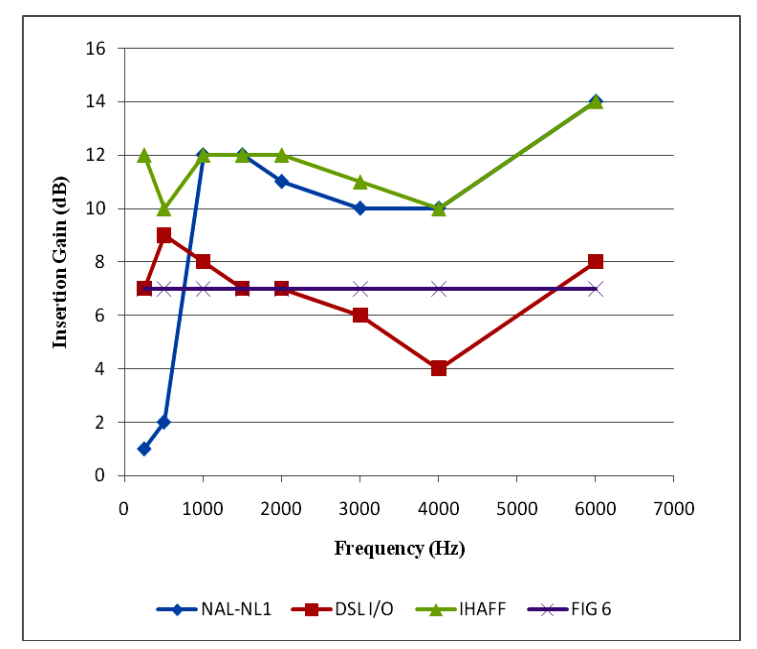

Figure 7c. Insertion gain at input level $80 \mathrm{~dB}$ SPL for each of the four selection procedures of a person with flat $40 \mathrm{~dB}$ hearing loss.
A system was developed, and used to test subjects with hearing loss of any category, and suggested suitable gain values for different octave frequencies, to obtain satisfaction among the hearing aid users. The gain suggestions were made based on the standard linear and non linear prescriptive procedures, such as NAL-R, POGO II, DSL, DSL I/O, IHAFF, NAL-NL1, NAL-NL2, and FIG6, depending on the type of hearing loss. Satisfaction among the hearing aid users was assessed using the speech discrimination score. Subjects with mild and moderate loss were satisfied with the suggestions made by either one of the prescriptive procedures from POGO II, NAL-R and DSL procedures. Subjects with sensorineural loss obtained satisfaction, only with suggestions made from any one of the non linear procedures such as NAL-NL1, NAL-NL2 and DSL I/O formulae. Speech intelligibility plays a vital role and has more value addition than speech audibility or loudness, for subjects with sensorineural hearing loss. Prescriptive procedures based on speech intelligibility like NAL-NL1 and NAL-NL2, provided successful gain suggestions than procedures based on loudness normalization. New hearing aid users required less target gain, than subjects who used hearing aids for more than two years. The system presented in this work is able to identify an appropriate prescriptive procedure for a specific type of audiogram, based on the trial results already obtained from the successful users of hearing aids. If the subjects are not satisfied with the gain suggestions made, an appropriate prescriptive procedure was suggested by the system, by which the subjects would be satisfied by adjusting the gain value by the audiologists. The suggested gain values are stored in the system. The stored data will be used in subsequent periods to derive a specific gain prescribing formula to suit our living conditions and languages.

\section{REFERENCES}

[1] Watson LA, Knudsen V. Selective amplification in hearing aids. J Acoust Soc of Am 1940; 18:401-8.

[2] Lybarger S . Method of fitting hearing aids . U .S . Patent Applications S .N . 543,278, July 3, 1944.

[3] Cornelisse LE, Seewald RC, Jamieson DG. The input output formula: a theoretical approach to the fitting of personal amplification devices. J Acoust Soc Am 1995; 97:1854-64.

[4] .Cox R, Goff CM, Martin SE, McLoud LL. The contour test: normative data. Presented at the American Academy of Audiology Convention ; 1994, Richmond, VA.

[5] Killion M. FIG6, Jackson Hole Rendezvous; 1994, Jackson, WY.

[6] Silman S, Gelfand SA, Siverman CA. Late onset auditory deprivation: effects of monaural versus binaural hearing aids. J Acoust Soc Am 1984; 76:1357-62.

[7] .Gelfand SA. Long-term recovery and no recovery from

\section{Conclusion}


auditory deprivation effect with binaural amplification: six cases . J Am Acad Audiol 1995; 6:141-9.

[8] .Hawkins D. Prescriptive approaches to selection of gain and frequency response, In : Mueller HG, Hawkins DB, Northern JL, editors . Probe tube microphone measurements: hearing aid selection and assessment. San Diego, CA: Singular Publishing; 1992.

[9] .Byrne D. Hearing aid gain and frequency response selection strategies. In: Studebaker GA, Hochberg I, editors. Acoustic factors affecting hearing aid performance. Boston : Allyn and Bacon; 1993

[10] Humes L, Hailing DC. Overview, rationale, and comparison of suprathreshold-based gain prescriptive methods In : Valente $M$, editor. Strategies for selecting and verifying hearing aid fittings. New York: Thieme-Stratton; 1994.

[11] McCandless G. Overview and rationale of threshold based hearing aid selection procedures. hl: Valente $\mathrm{M}$, editor. Strategies for selecting and verifying hearing aid fittings, New York: Thieme-Stratton; 1994.

[12] McCandless G. (1988) . Hearing aid formulae and their application . In : Sandlin R, editor. Hearing aid handbook : volume I theoretical and technical considerations . San Diego, CA: College-Hill Press ; 1994.

[13] Cox R. Using loudness data for hearing aid selection: the IHAFF approach . Hear J $1995 ; 48: 2,10,39-44$.

[14] IHAFF. A comprehensive hearing aid fitting protocol, distributed at Jackson Hole Rendezvous ; 1994, Jackson, WY.

[15] Killion M . Talking hair cells: what they say about hearing aids. In : Berlin CI. editor . Hair cells \& hearing aids, San Diego, CA: Singular Publishing ; 1995.

[16] Dillon H (1999). NAL-NL1: A new prescriptive fitting procedure for non-linear hearing aids. Hearing Journal, 52(4):10-16.

[17] Dillon H, Keidser G, Ching T, Flax $\mathrm{M}$ and Brewer $\mathrm{S}$. Development of the NAL-NL2. XIX Australian Audiological Society National Conference, Sydney, May 2010.

[18] Byrne D, Tonnisson W. Selecting gain of hearing aids for persons with sensorineural hearing impairments. Scand Audiol $1976 ; 5$ :51-9.

[19] Byrne D, Dillion H. The National Acoustic Laboratories (NAL) new procedure for selecting the gain and frequency response of a hearing aid. Ear Hear 1986 ; : 257-65.

[20] Mueller HG . Individualizing the ordering of custom hearing aids, In : Mueller HG, Hawkins DB, Northern JL, editors. Probe-microphone measurements : hearing aid selection and assessment, San Diego, CA : Singular Publications ; 1992.

[21] Bryne D, Parkinson A, Newell P. Hearing aid gain and frequency response requirements for the severely/profoundly hearing impaired. Ear Hear $1990 ; 11: 40-9$.

[22] Byrne D, Parkinson A, Newell P. Modified hearing aid selection procedures for severe/profound hearing losses . In : Stude baker G, Bess F, Beck L, editors . The Vanderbilt hearing aid report II . Parton, MD : York Press ; 1991 . p . 295-300.

[23] McCandless G . Lyregaard P. Prescription of gain and output (POGO) for hearing aids . Hear Instr 1983 ;34 :16-21.

[24] Schwartz D, Lyregaard P, Lundh P. Hearing aid selection for severe- to-profound hearing loss . Hear J 1988; 41:13-7.

[25] Seewald RC, Ross M, Spiro MK . Selecting amplifications characteristics for young hearing-impaired children, Ear Hear $1985 ; 6: 48-51$.

[26] Seewald RC, Ramji KV, Sinclair ST, Moodie KS, Jamieson DG. User's manual : computer assisted implementation of the desired sensation level method for electroacoustic selection and firring in children. Version 3.1 , London : University of Western Ontario; 1993.

[27] Van Vliet D . Determining contour loudness judgements . Hear Instr 1995 ;46(March) :30.

[28] Killion M, Fikret-Pasa S . The three types of sensorineural hearing loss : loudness and intelligibility consideration. Hear J 1993 ;46 :31-6.

[29] Hawkins D . Corrections and transformations relevant to hearing aid selection. In : Mueller HG, Hawkins, DB, Northern JL, editors . Probe tube microphone measurements : hearing aid selection and assessment. San Diego, CA : Singular Publishing; 1992.

[30] Killion M, Revit L . CORFIG and GIFROC. In : Studebaker GA, Hochberg I, editors . Acoustic factors affecting hearing aid performance . Boston, MA : Allyn and Bacon ; 1993.

[31] Byrne D, Dillon H, Ching T, Katsch R and Keidser G (2001). NAL-NL1 procedure for fitting nonlinear hearing aids: characteristics and comparisons with other procedures. Journal of the American Academy of Audiology, 12:37-51.

[32] Keidser G and Dillon H (2007). What's new in prescriptive fittings Down Under? In Seewald R (Ed.), Hearing Care for Adults 2006. (pp.133-142).

[33] Moore, B.C.J., Glasberg, B.R. 1997. A model of loudness perception applied to cochlear hearing loss. Aud Neurosci 3, 289-311.

[34] Moore, B.C.J., Glasberg, B.R. 2004. A revised model of loudness perception applied to cochlear hearing loss. Hear Res 188,70-88.

[35] Keidser G, Dillon H, Dyrlund O, Carter L and Hartley D (2007). Preferred compression ratios in the low and high frequencies by the moderately severe to severe-profound population. Journal of the American Academy of Audiology, 18(1):17-33.

[36] G. Keidser $\mid$ H.R. Dillon $\mid$ M. Flax $\mid$ T. Ching $\mid$ S. Brewer (2011)Journal: Audiology Research ISSN 2039-4330 Volume: 1; Issue: 1; Start page: e24. 